\title{
A modeling study of idiopathic intracranial hypertension
}

\author{
S. A. Stevens ${ }^{1}$, W. D. Lakin ${ }^{2}$, N. J. Thakore ${ }^{3}$, P. L. Penar ${ }^{2}$ \\ \& B. I. Tranmer ${ }^{2}$ \\ ${ }^{1}$ School of Science, Penn State Erie, The Behrend College, U.S.A. \\ ${ }^{2}$ The University of Vermont, U.S.A. \\ ${ }^{3}$ MetroHealth Medical Center and Case Western Reserve University, \\ U.S.A.
}

\begin{abstract}
Idiopathic intracranial hypertension (IIH) is a syndrome of unknown cause characterized by elevated intracranial pressure (ICP). A stenosis of the transverse sinus is observed in many patients suffering from IIH. The role that this feature plays in the etiology of IIH remains unresolved. It may be the primary cause of IIH, an exacerbating factor, or merely a secondary event. A lumped-parameter model of intracranial pressure dynamics has been modified to accommodate transverse sinus stenosis via a Starling-like resistor. In the absence of this type of resistor the system has a unique asymptotically-stable steady-state with normal pressures. With this type of resistor present, a second, asymptotically-stable steady-state may exist. This state is characterized by elevated ICP concurrent with a compressed transverse sinus. It is hypothesized that IIH is a physiological manifestation of this elevated steady state. We conclude that the primary cause of IIH is a compressible, as opposed to rigid, transverse sinus, and that the observed stenosis is a necessary characteristic of the elevated steady state. Simulations suggest possible diagnostic techniques and comparisons are made to clinical data.
\end{abstract}

\section{Introduction}

Idiopathic intracranial hypertension (IIH), also called pseudotumor cerebri and benign intracranial hypertension, is a syndrome of unknown cause characterized by elevated intracranial pressure (ICP) without evidence of ventricular dilatation, mass lesion, cerebrospinal fluid (CSF) abnormality, or dural sinus thrombosis. It 
presents with symptoms of headache, nausea, vomiting, papilledema, and visual obscurations [1]. In many patients suffering from IIH, a stenosis or tapering of the transverse sinuses is observed by magnetic resonance venography or retrograde catheter venography $[2,3,4]$. The role played by transverse sinus stenosis in the etiology of IIH has been unresolved and is a major focus of the present study.

A lumped-parameter model of intracranial pressure dynamics is utilized to explore the relationship between IIH and transverse sinus stenosis. In previous models of this type $[5,6,7,8]$ the sinuses have been considered rigid so as to withstand the negative transmural pressure normally endured by these vessels. In the present work, the model in [7] has been modified so that this assumption is no longer made. Specifically, the resistance to venous sinus drainage is formulated to be sensitive to transmural pressure changes. This modification now allows for partial collapse of the transverse sinuses in the face of elevated ICP with a resulting increase in resistance to venous sinus drainage.

\section{The lumped parameter model}

A diagram of the lumped-parameter model that has been enhanced for this study is shown in Figure 1. Previously, this model has been used to study steady-state intracranial pressures in microgravity [7]. A brief description of the model is given below. A full description that includes calibration of the normal parameter values is given in [7].

\subsection{Features of the mathematical model}

Cerebral blood flow $\left(Q_{I C}\right)$ and CSF formation by the choroid plexus $\left(Q_{C F}\right)$ are considered constant in the current application. Filtration from the intracranial capillaries across the blood-brain barrier $\left(Q_{C B}\right)$ is modeled by the Starling-Landis equation involving both hydrostatic and osmotic forces. All other flows are related to pressure differences by

$$
Q_{i j}=\left(P_{i}-P_{j}\right) / R_{i j}=Z_{i j}\left(P_{i}-P_{j}\right),
$$

where $Q_{i j}$ is the flow from compartment $i$ to compartment $j, P_{i}$ and $P_{j}$ are the spatially-averaged pressures of compartments $i$ and $j$ respectively, $R_{i j}$ is the lumped resistance, $R_{i j}=-R_{j i}$, and $Z_{i j}$ is the fluidity (inverse of $R_{i j}$ ). Volume adjustments between adjacent compartments are related to changes in pressures by local compliance parameters $C_{i j}=C_{j i}$. Applying the law of conservation of mass in compartments $\mathrm{I}, \mathrm{C}, \mathrm{S}, \mathrm{F}, \mathrm{B}$, and $\mathrm{T}$ results in a set of governing differential equations defined in matrix form by

$$
C \frac{d P}{d t}+Z P=Q,
$$

where $P=\left[P_{I}, P_{C}, P_{S}, P_{F}, P_{B}, P_{T}\right]^{t r}, C$ is a compliance matrix, $Z$ is a fluidity matrix, and $Q$ is a vector of forcing terms that involves extra-cranial pressures and 


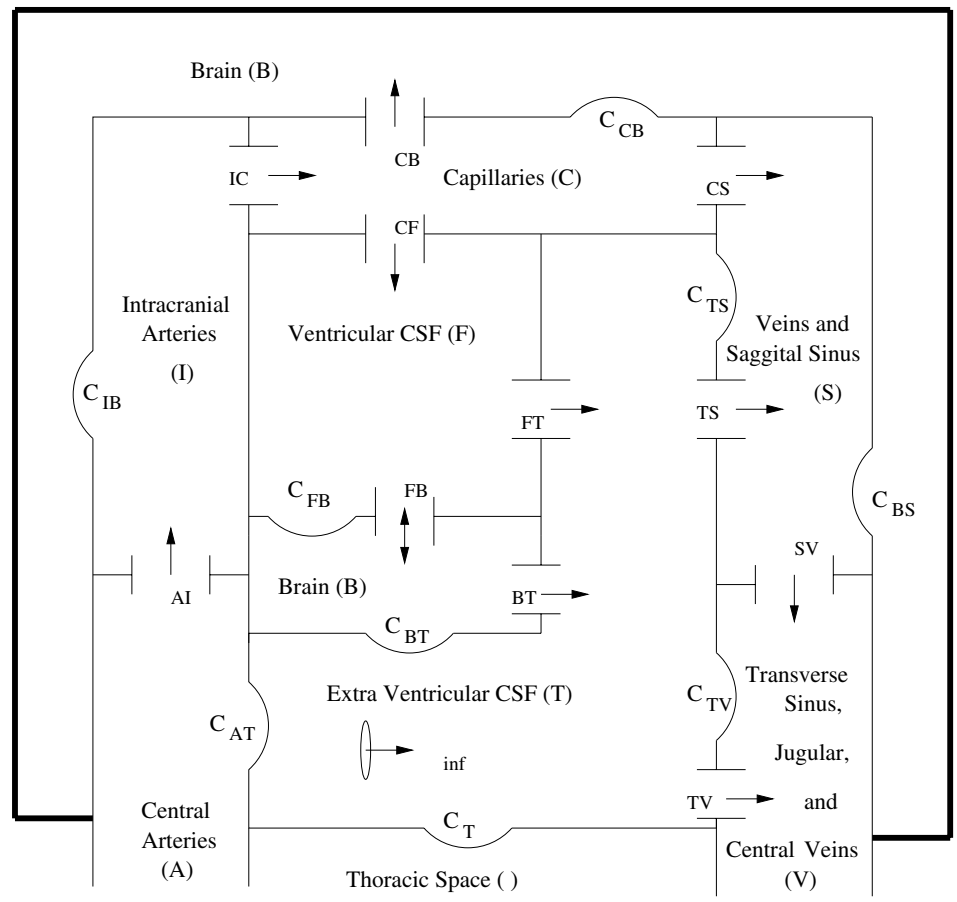

Figure 1: A diagram of the lumped-parameter model. The dark line represents the rigid cranial wall. $Q_{i j}$ represents fluid flow from compartment $i$ to compartment $j$. Arrows indicate the customary direction of flow. $Q_{\text {inf }}$ represents an infusion rate of CSF. $C_{i j}$ represents a distensible surface between compartments $i$ and $j$. The Starling-like resistor described in this paper is associated with $Q_{S V}$.

known flows. The matrix equation (2) may appear to be linear, but entries in the compliance and fluidity matrices will be functions of compartmental pressures and time, introducing nonlinearities.

If the oscillatory effects of the forcing terms in $Q$ are subtracted, the solution of (2) is a set of time-dependent intracranial pressures averaged over each cardiac cycle. It was shown [7] that if the fluidity terms in $Z$ are all constants then all solutions of (2) will tend to a unique steady state. For the current investigation,the fluidity term $Z_{S V}$ is no longer constant but is allowed to be pressure sensitive. With this modification, convergence to a unique steady-steady state is no longer guaranteed. Indeed, analysis of the various possible steady-states and their stability properties constitutes a major portion of the mathematics involved in this work. 


\subsection{The Starling-like resistor}

A Starling resistor in a model for fluid flow through a collapsible vessel is a resistance term that is dependent upon transmural pressure. When transmural pressure is large, the vessel is considered open and the resistance to flow is small. Conversely, if the transmural pressure is small or negative, then the vessel is partially or completely collapsed resulting in a large (or possibly infinite) resistance. In many models $[8,9,10]$, a Starling resistor has been placed at the location where the cerebral veins empty into the saggital sinus near the cerebral lacunae. Further downstream, Pedley et al. [11] suggest a similar phenomenon in the jugular vein of the giraffe. A common feature of these locations is that the transmural pressure is positive in the normal resting state. Starling resistors have not previously been introduced into the venous sinuses because normal transmural pressure in these vessels is negative, and rigid walls were assumed to prevent collapse.

The observed stenosis of the transverse sinuses in IIH patients suggests that the venous sinuses are not completely rigid. Therefore, to allow for collapse we will introduce a Starling-like resistor dependent on transmural pressure at the transverse sinus level of the model to represent the resistance to flow from compartment $\mathrm{S}$ to $\mathrm{V}$. The traditional form of a Starling resistor does not apply at this point in the vasculature as the normal transmural pressure is negative, so the Starlinglike resistor for the fluidity $Z_{S V}=1 / R_{S V}$ will be defined in such a way that this fluidity term is positive despite the transmural pressure $P_{V}-P_{T}$ being negative. However $Z_{S V}$ will decrease (resistance will increase) when $P_{V}-P_{T}$ becomes more negative. In particular, $Z_{S V}$ is defined by

$$
Z_{S V}=\operatorname{Max}\left[\bar{Z}_{S V}\left(1-m\left(P_{T V}-\bar{P}_{T V}\right)\right), p \bar{Z}_{S V}\right] \quad 0 \leq p \leq 1
$$

Here, $P_{T V}=P_{T}-P_{V}$, an overbar indicates the normal resting value, and Max refers to the larger of the two terms. The graph of the relationship between $Z_{S V}$ and $P_{T V}$ is piecewise-linear and continuous with an initial decreasing straight line segment of slope $-m \bar{Z}_{S V}$ that passes through the scale point $\left(\bar{P}_{T V}, \bar{Z}_{S V}\right)$, calibrated as in [7]. From equation (3), $p \bar{Z}_{S V}$ is the minimum value of $\bar{Z}_{S V}$, and beyond the pressure difference $P_{T V}$ that produces this minimum the graph of $Z_{S V}$ is a horizontal line at this minimum value. It may well be the case that in healthy individuals no such Starling-like resistor is present and the sinuses are indeed rigid. This is achieved in the model by setting $m=0$ and the fluidity is therefore constant.

A minimum on $Z_{S V}$ in equation (3) is imposed for three reasons. First, fluidity cannot be negative. Second, even in the face of ICP equal to arterial pressure there was only an $80 \%$ reduction in cerebral blood flow according to the model presented by Tym et al. [12]. Finally, there may be other pathways for venous drainage [13].

Two unique features of this Starling-like resistor should be noted. First, as discussed above, the transmural pressure is negative in the normal state. A second unique feature is that this resistor is based on the transmural pressure in a downstream compartment $V$ as opposed to the upstream compartment $S$. One reason 
for this choice is that the resistance $\left(R_{S V}\right)$ in a lumped parameter model represents the total resistance between pressure reference points $P_{S}$ and $P_{V}$. Therefore some of the resistance due to flow through compartment $V$ is represented by $R_{S V}$. Further, it has been shown [14] that when a compliant vessel with internal flow starts to collapse due to a uniformly applied external pressure, it does so at the furthest downstream location. When this occurs, the change in total resistance is due to the collapse at this point, and the degree of this collapse will be dependent upon the difference between the external and downstream pressures.

\section{Steady state analysis}

The complexity and the number of equations involved in the current model make analytic results for potential steady states nearly impossible to obtain. Rigorous analysis of an analogous two compartment model was used to guide numerical results for the current model to obtain the conclusions presented in this section.

With the introduction of a pressure sensitive fluidity term $Z_{S V}$, a steady state, or equilibrium solution, to equation (2) is no longer constrained to be unique, and the number of steady state solutions be may increase from unity, depending on the values of the parameters $m$ and $p$ in equation (3). An increasing straight line

$$
m=\alpha \bar{Z}_{S V}\left(\bar{Q}_{S V}\right)^{-1} p+\bar{Q}_{F V}\left(\bar{Q}_{S V} \bar{P}_{S V}\right)^{-1} \text { where } \alpha=1+Z_{F V}\left(Z_{F S}\right)^{-1}
$$

now divides the $m-p$ parameter plane into regions. Below this line there is one steady state and above it there are three. In the region with one steady state, that unique state is represented by a stable node in phase space that is associated with normal pressures. In the region with three steady states, one state is the original stable node of normal pressures, a second state is an unstable saddle point of elevated pressures, and the third state is another stable node with elevated pressures. Thus the dividing line in the $m$ - $p$ parameter plane is a saddle-node bifurcation curve.

This situation is clearly seen in Figure 2, which gives an example of the $P_{S^{-}}$ $P_{T}$ phase plane. When there are three steady states the two unique trajectories that lead to the unstable saddle point delineate the basins of attraction to the two stable nodes. Other than the two theoretical trajectories that lead to the saddle point, all other trajectories will tend to one or the other of the two stable states, so convergence to the saddle point is never realized in practice. The three points lie in a line, and the value of $p$ determines the location of the stable node while the value of $m$ determines the location of the saddle point. As $p$ decreases, the stable node with elevated pressures moves up and to the right. Therefore the magnitude of the pressures in the elevated state is determined by $p$. As $m$ decreases, the saddle point moves up and to the right as well. Therefore, as $m$ decreases so does the likelihood of a transition from the base-value state to the elevated-state. 


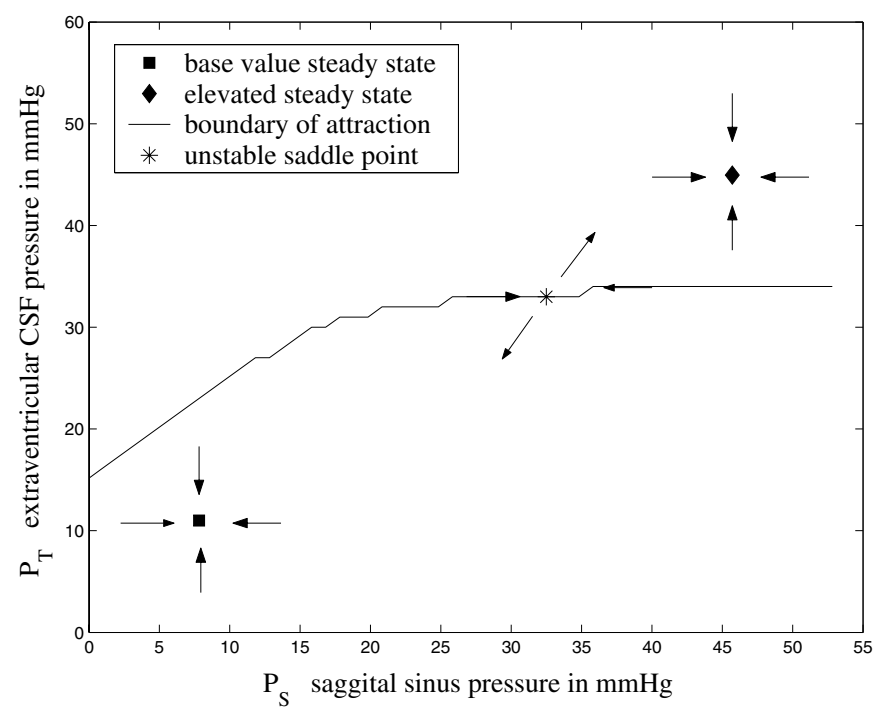

Figure 2: The $P_{s}-P_{t}$ phase plane portrait in the case of three steady states. Here, $p=0.06$ and $m=0.04$ and all other parameter values are taken from [7].

\section{Time dependent simulations of CSF infusion/withdrawal}

The current model was used to study the response of lumbar CSF pressure to CSF infusion and withdrawal. In the case of a temporary infusion, the initial state was taken as the stable base state with normal pressures, and simulations with the Starling-like resistor both present and absent were run. These time-dependent simulations indicate that a rapid transition from a normal to an elevated steady state in response to such an infusion is a key predictor of the presence of a compressible transverse sinus and susceptibility to IIH. The present infusion results are illustrated in Figure 3 where the solid curve describes the response of $P_{T}$ when a Starling-like resistor is present and the dashed curve depicts the predicted response to an identical infusion when no Starling-like resistor is present, i.e. $m=0$ in equation (3). These simulation results are in full accord with the clinical findings of Higgins and Pickard [3] who reported that after an IIH patient was successfully treated with a shunt implant, the patient's CSF pressure still exhibited a "rapid" increase from normal in response to infusion.

For the time dependent simulations in the complementary case of a temporary CSF withdrawal, typical results are depicted in Figure 4 which compares the responses of $P_{T}$ and $P_{S}$. A Starling-like resistor is assumed present in these simulations, and indicative of IIH the initial state was taken to be the stable elevated pressure state for the chosen parameter values. A sharp transition drop in pressures is again observed in these simulations, although at the termination of the 
withdrawal $P_{T}$ and $P_{S}$ tend to the normal pressure state at different rates. As seen in Figure 4, the saggital sinus pressure rapidly assumes its normal value while the lumbar CSF pressure undershoots its normal value and then has a slow return toward normal CSF pressure.

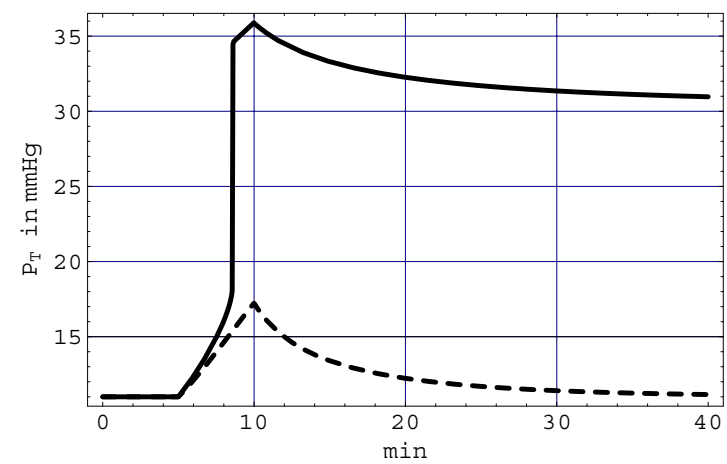

Figure 3: The simulated CSF pressure $\left(P_{T}\right.$ in $\left.\mathrm{mmHg}\right)$ response to an infusion rate of $2 \mathrm{ml} / \mathrm{min}$ for 5 minutes starting at $t=5$ minutes. Solid: a Starling-like resistor with $p=0.1$ and $m=0.08$. Dashed: no Starling-like resistor.

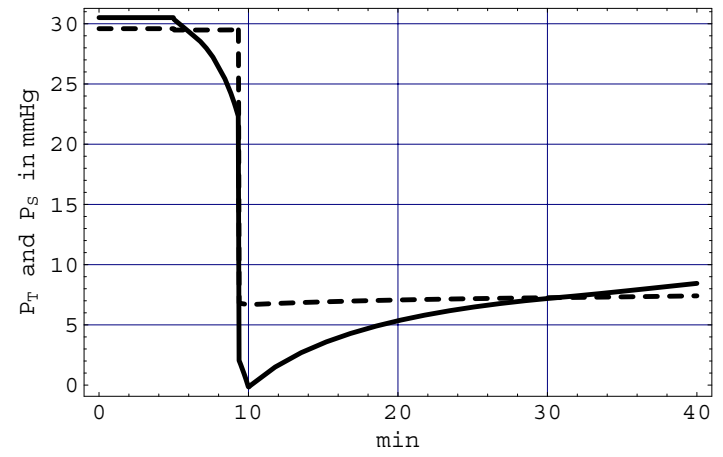

Figure 4: The simulated CSF (solid) and saggital sinus (dashed) pressure response to a withdrawal rate of $5 \mathrm{ml} / \mathrm{min}$ for 5 minutes starting at $t=5$ minutes. In both cases there is a Starling-like resistor with $p=0.1$ and $m=0.08$ starting at the elevated state. 


\section{Comparisons with clinical data}

As an indication of the ability of a Starling-like resistor located at the transverse sinus to explain aspects of IIH, it will be demonstrated that the reported clinical observations of King et al. [4] for IIH patients can be interpreted by using the present model with appropriate calculated values for the resistor parameter $p$ and the scale pressure $\bar{P}_{T}$. In the comparison simulations, the value of the parameter $m$ is assumed sufficiently large that, consistent with the measured data, an elevated steady state exists.

We will consider the three cases in [4] where CSF and various venous pressures were measured before and after a 20 to $25 \mathrm{ml} \mathrm{CSF}$ withdrawal. This data is given in the first 6 columns of Table 1. The data measured prior to CSF withdrawal is assumed to be that of an elevated IIH pressure state and is indicated by a superscripted asterisk. The results depicted in Figure 4 indicate that the CSF pressure response after the withdrawal will drop below the normal base value while the saggital sinus pressure after the withdrawal will be indicative of the actual base-value state. It is therefore assumed that the base-value state of the saggital sinus pressure is that measured immediately after the CSF withdrawal, and this is denoted by an overbar. This value is then used to estimate the value of $p$ in the Starling-like resistor under the assumption that CSF production and cerebral blood flow remain constant. The estimated value of $p$ is given in the seventh column of Table 1 . The normal base value for the lumbar CSF pressure $\bar{P}_{T}$, displayed in the eighth column of Table 1, is then predicted to achieve the observed elevated state in columns 1 through 3. For Patient 2 in row 2 of Table 1, no value for $\bar{P}_{T}$ could be calculated to give the observed elevated pressure state unless the percentage of CSF production normally absorbed into the saggital sinus was reduced from $75 \%$ [15] to $50 \%$. When this adjustment was made, a base value CSF pressure of $12.85 \mathrm{mmHg}$ was estimated.

Row 1 of Table 1 shows that for Patient 1 the CSF pressure after the withdrawal is well below the predicted normal base CSF pressure. It appears that this measurement was taken immediately after the termination of the large withdrawal and reflects the well value of the previously-noted undershoot of the normal base value $\bar{P}_{T}$ by $P_{T}$. By contrast, the measured values of $P_{T}$ and the predicted normal values $\bar{P}_{T}$ for Patients 2 and 3 are relatively close indicating that these measurements were probably taken at a time beyond the undershoot when $P_{T}$ was approaching its normal base value.

\section{Discussion}

In many mathematical models of intracranial blood flow, a Starling or Starling-like resistor is assumed to exist at the location of collapsible veins $[8,9,10,11]$ where the normal transmural pressure is positive. At the location of the transverse sinus, however, the normal transmural pressure is negative, and so these vessels have traditionally been considered rigid leading to resistance (or fluidity) terms that are assumed constant. In order to accommodate the observed stenosis of these vessels 
Table 1: Data from three IIH patients [4] before and after a 20-25 ml CSF withdrawal and the resulting parameter assignments to the model. Pressures are in $\mathrm{mmHg}$.

\begin{tabular}{|c|c|c||c|c|c||c|c|}
\hline \multicolumn{3}{|c||}{ Before withdrawal } & \multicolumn{3}{c||}{ After withdrawal } & Parameter Assignments \\
\hline$P_{T}^{*}$ & $P_{S}^{*}$ & $P_{V}^{*}$ & $P_{T}$ & $\bar{P}_{S}$ & $\bar{P}_{V}$ & $p$ & $\bar{P}_{T}$ \\
\hline \hline 33.1 & 20 & 4 & 3.7 & 2 & 0 & 0.125 & 16.1 \\
\hline 29.4 & 35 & 6 & 12.5 & 9 & 8 & 0.034 & 12.85 \\
\hline 26.5 & 20 & 6 & 19.1 & 14 & 5 & 0.643 & 20.8 \\
\hline
\end{tabular}

in IIH, a Starling-like resistor has been introduced into the present mathematical model at the location of the transverse sinus. The variation of this resistor is based on the transmural pressure difference just downstream from the collapse point.

A steady-state analysis of the current model demonstrates that when collapse of the transverse sinus is allowed, an additional stable steady-state with elevated pressures may be present as a solution of the system. It is hypothesized that IIH is a physiological manifestation of this additional stable elevated pressure state, and that a requirement for the development of IIH is a collapsible, as opposed to fully rigid, transverse sinus. Temporal simulations suggest that in clinical data rapid transitions between normal and elevated states in response to CSF infusions or withdrawals are indicative of the presence of such collapsible vessels in a subject. A notable finding is that there appears not to be a tight range for the parameter $p$ in equation (3) associated with potential IIH. When this parameter was calculated from the limited data in Table 1 the values of $p$ ranged from .034 to .643. Additional measured data from IIH patients is required to fully validate the present hypotheses.

\section{References}

[1] Binder, D., Horton, J., Lawton, M. \& McDermott, M., Idiopathic intracranial hypertension. Neurosurgery, 54(3), pp. 538-540, 2004.

[2] Farb, R., Vanek, I., Scott, J., Mikulis, D., Willinsky, R., Tomlinson, G. \& terBrugge, K., Idiopathic intracranial hypertension: The prevalence and morphology of sinovenous stenosis. Neurology, 60, pp. 1418-1424, 2003.

[3] Higgins, J. \& Pickard, J., Lateral sinus stenosis in idiopathic intracranial hypertension resolving after csf diversion. Neurology, 62, pp. 1907-1908, 2004.

[4] King, J., Mitchell, P., Thomson, K. \& Tress, B., Manometry combined with cervical puncture in idiopathic intracranial hypertension. Neurology, 58, pp. 26-30, 2003. 
[5] Sorek, S., Bear, J. \& Karni, Z., A non-steady compartmental flow model of the cerebrovascular system. J Biomechanics, 21, pp. 695-704, 1988.

[6] Stevens, S. \& Lakin, W., Local compliance effects on the global CSF pressure-volume relationship in models of intracranial pressure dynamics. Mathematical and Computer Modelling of Dynamical Systems, 6(4), pp. 445-465, 2001.

[7] Stevens, S., Lakin, W. \& Penar, P., Modeling steady-state intracranial pressures in supine, head-down tilt, and microgravity conditions. Aviat Space Environ Med, 76, pp. 329-338, 2005.

[8] Ursino, M., A mathematical study of human intracranial hydrodynamics. part 1 - the cerebrospinal fluid pulse pressure. Annals of Biomedical Engineering, 16, pp. 379-401, 1988.

[9] Czosnyka, M., Piechnik, S., Richards, S., Kirkpatrick, P., Smielewski, P. \& Pickard, J., Contribution of mathematical modelling to the interpretation of bedside tests of cerebrovascular autoregulation. J Neurol Neurosurg Psychiatry, 63, pp. 721-731, 1997.

[10] Piechnik, S., Czosnyka, M., Richards, H., Whitfield, P. \& Pickard, J., Cerebral venous blood outflow: A theoretical model based on laboratory simulation. Neurosurgery, 49, pp. 1214-23, 2001.

[11] Pedley, T., Brook, B. \& Seymour, R., Blood pressure and flow rate in the giraffe jugular vein. Philosophical Transactions: Biological Sciences, 351(1342), pp. 855-866, 1996.

[12] Tym, R., Lichtenstein, S. \& Leutheusser, J., The monroe-kellie doctrine and the intracranial venous space at the limit of raised intracranial pressure: An hydrodynamic experimental approach. Intracranial Pressure: Experimental and Clinical Aspects, eds. M. Brock \& H. Dietz, Springer-Verlag: Berlin, pp. 139-143, 1972.

[13] Bateman, G., Vascular hydraulics associated with idiopathic and secondary intracranial hypertension. Am J Neuroradiol, 23, pp. 1180-1186, 2002.

[14] Grotberg, J. \& Jensen, O., Biofluid mechanics in flexible tubes. Annu Rev Fluid Mech, 36(5), pp. 121-47, 2004.

[15] Bozanovic-Sosic, R., Mollanji, R. \& Johnston, M., Spinal and cranial contributions to total cerebrospinal fluid transport. Am J Regul Physiol Integr Comp Physiol, 281(3), pp. R909-R916, 2001. 